\section{Problem Solving Heuristics, Affect, and Discrete Mathematics}

\author{
Gerald A. Goldin, New Brunswick, NJ (USA)
}

\begin{abstract}
It has been suggested that activities in discrete mathematics allow a kind of new beginning for students and teachers. Students who have been "turned off" by traditional school mathematics, and teachers who have long ago routinized their instruction, can find in the domain of discrete mathematics opportunities for mathematical discovery and interesting, nonroutine problem solving. Sometimes formerly low-achieving students demonstrate mathematical abilities their teachers did not know they had. To take maximum advantage of these possibilities, it is important to know what kinds of thinking during problem solving can be naturally evoked by discrete mathematical situations - so that in developing a curriculum, the objectives can include pathways to desired mathematical reasoning processes. This article discusses some of these ways of thinking, with special attention to the idea of "modeling the general on the particular." Some comments are also offered about students' possible affective pathways and structures.
\end{abstract}

Kurzreferat: Vielfach wird vorgeschlagen, dass Inhalte der Diskreten Mathematik geeignet sind, Lehrern und Schülern ein neues Bild der Mathematik zu vermitteln. Mathematische Entdeckungen an Problemen, die nicht zur Unterrichtsroutine gehören, sind hier leichter möglich als in vielen anderen Gebieten der Mathematik. Das gilt selbst für Schülerinnen und Schüler, die eher als weniger leistungsstark angesehen werden können. Damit Lehrerinnen und Lehrer allerdings die möglichen Vorteile optimal nutzen können, sollten sie wissen, welche Art von Denken und mathematischer Argumentation bei solchen Aufgaben gefordert ist. Der Artikel diskutiert das Thema und geht dabei insbesondere auf das Modellieren des allgemeinen Falls auf der Basis eines speziellen Falls ein. Einige Bemerkungen befassen sich mit der affektiven Komponente des Problemlösens.

\section{ZDM-Classifikation: C20, C30, D50, N70}

\section{Possibilities for Discrete Mathematics}

In their accompanying article, DeBellis and Rosenstein (2004) describe a vision for discrete mathematics in the schools of the United States, one toward which they have both contributed substantially over a decade and a half. They see the domain of discrete mathematics - a looselydefined term that includes combinatorics, vertex-edge graphs, iteration and recursion, and many other topicsas providing teachers with "a new way to think about traditional mathematical topics and a new strategy for engaging their students in the study of mathematics." Through experiences in discrete mathematics, they suggest that teachers may better be able to help children "think critically, solve problems, and make decisions using mathematical reasoning and strategies." And they cite Gardiner (1991) in cautioning, "If instead discrete ma- thematics is introduced in the schools as a set of facts to be memorized and strategies to be applied routinely ... [its qualities] as an arena for problem solving, reasoning, and experimentation are of course destroyed."

It is certainly an appealing idea that students who have been "turned off" by traditional school mathematics, and teachers who have long ago routinized their instruction, can find something quite new here. Evidently the novel possibilities have to do less with particular formulas and techniques of combinatorics, search or sorting algorithms, theorems about graphs, and so on, than with the opportunities for interesting, nonroutine problem solving and for mathematical discovery that discrete mathematics may provide (cf. Kenney \& Hirsch, 1991; cf. Rosenstein, Franzblau, \& Roberts, 1997). Here I would like to emphasize the importance of characterizing these opportunities more specifically, both mathematically and cognitively.

The following questions deserve consideration: (1) What especially desirable ways of thinking, powerful problem-solving processes, or other important mathematical competencies do discrete mathematical situations naturally evoke? (2) Why are these particular processes or capabilities evoked in students? Under what problem conditions do we expect them to occur? Why might we anticipate the emergence of previously hidden mathematical capabilities in some students, and which capabilities are these? (3) How can we consciously structure students' activities so as to best encourage the further development of the mathematical capabilities we have identified? Can we do this through discrete mathematics more readily or naturally than we could through a comparable commitment to conventional arithmetic, algebra, or geometry? (4) How can or should we assess the degree to which student performance is enhanced-in discrete mathematics particularly, and in the mathematical field generally?

Of course, in a short article one can discuss only small parts of these questions. Here I shall focus on just two aspects: (a) heuristic processes for mathematical problem solving, especially the way of thinking we may call "modeling the general on the particular", and (b) students' affective pathways and structures.

\section{A Problem for Discussion}

Let us consider a specific and rather well-known "nonroutine" problem-solving activity, in order to lend concreteness to the discussion.

You are standing on the bank of a river with two pails. One pail holds exactly 3 liters of water, and the other holds exactly 5 liters. The pails are not marked for measurement in any other way. How can you carry exactly 4 liters of water away from the river?

This is a problem I have given many times, to children and to adults. While it does not fall clearly into one of the above-mentioned domains of discrete mathematics, it has features in common with problem activities drawn from many of those domains. It is "discrete" in the sense of involving discrete steps that are permitted at any point by the problem conditions. It is sufficiently "nonroutine" for 
even the mathematical interpretation of the problem conditions to be challenging to many. Like "counting problems" in combinatorics, it requires the problem solver to devise some means of keeping track of what has already been done. Like "coloring problems" and "shortest route" problems, it invites successive trials that may fail to satisfy the problem conditions. Like many problems in discrete mathematics, this problem is also suggestive of a possible hidden structure that would, if recognized, make the solution easy to see.

Schematically, one may represent this problem by means of a state-space diagram as in Fig. 1, where each node (or problem state) corresponds to a configuration with a fixed, known volume of water in each of the pails, and each arc corresponds to the step of filling a pail at the river, emptying a pail, or pouring water from one pail into the other until the latter is full (Goldin,1984). In Fig. 1 the ordered pair of numbers $(0,2)$, for example, refers to the configuration where the 3-liter pail is empty and there are 2 liters of water in the 5-liter pail.

Fig. 1. Schematic representation of paths through the state-space for the problem of the two pails

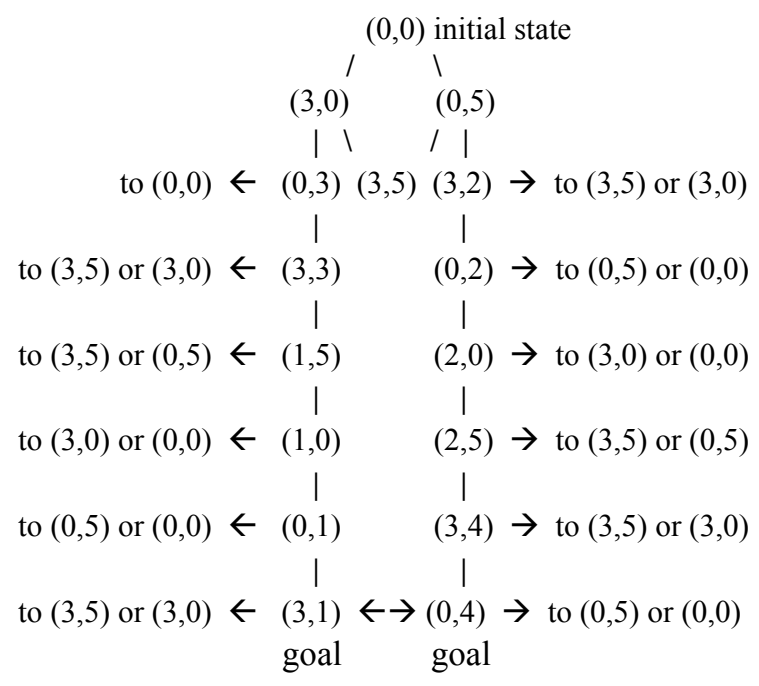

In effect, such a representation is a directed, connected vertex-edge graph, where a selected vertex (node) is understood to be the "initial state" and a characterization is provided of vertices (nodes) that are "goal states." The problem is thus one of finding a path along directed edges through this graph, linking the initial state to one of the goal states. We see-rather trivially, once the graph has been displayed - that there are two such solution paths, that they are related by symmetry but not equivalent at the level of pails and moves, and that each is only seven steps long. Furthermore, we note that there is in an important sense no way to go wrong in searching this statespace. That is, if one begins with the empty pails and takes any step at all, and then just proceeds without giving up or returning to one of the initial few states, one inevitably reaches a goal state. There are no "blind alleys" in this particular directed graph.

Why, then, is a strong sense of impasse experienced by some who attempt this problem? What are the desirable processes that this problem has a high likelihood of evoking, and why are they likely?
Of course, the problem solver does not have in front of her the representation of Fig. 1. The first challenge is to interpret the problem statement in a way that makes conventional "mathematical sense," turning the problem into one where something specific and non-arbitrary can be done in the face of requirements that may superficially appear to describe an impossibility. Thus some solvers respond initially with ideas that fall outside the problem conditions. Common initial responses include, "two liters in each pail" (without suggesting a way of arriving at such a configuration), or "three and five are eight, and four is half of eight, so I'll fill each pail halfway" (without attending to the condition that the pails are unmarked for measurement).

Naturally such answers do make sense. The fact that they are outside the intention behind the stated problem conditions does not mean they are undesirable - indeed, thinking "outside the box" is often just what non-routine problems require. Here, further questions or elaboration by the one who posed the problem can suggest that the problem is not yet solved: "How can you obtain two liters in each pail?" or "There is no way to fill the pails exactly halfway, when they aren't marked for measurement." An early point of possible impasse, then, occurs with the necessity of realizing that not only may either pail be filled to the brim at the river bank with knowledge of the volume, but also (more profoundly) that the contents of one pail may be partially poured into the other pail until the latter is full, allowing knowledge of the volume of water remaining.

Supposing these possibilities to be understood-i.e., adequately represented internally-by the problem solver, important potential impasses still remain. Many solvers begin to imagine pouring water from pail to pail, but after three or four steps come to feel they are making no progress - and repeatedly start over. Some are hesitant to construct an external written record, or perhaps are not sure how to do it-but without some systematic external representation, the memory load is high. "I forgot where I was", "I'm not getting anywhere", or "I'm sure I did this before, and it didn't work" are typical comments, even when the potential solver is only one step away from the goal. Some give up in frustration after a short series of such attempts. Others overcome this impasse by keeping track systematically of the steps they have taken, or by persevering despite feelings of "getting nowhere."

One possible explanation for this phenomenon is the absence of an obvious evaluation criterion whereby an intermediate state - $(2,5)$ for instance - can be judged as "closer" to the goal of having 4 liters than a state such as $(3,2)$ reached three steps earlier. Most problem solvers do try to avoid returning to states reached before, and if enforced rigorously, this criterion alone is sufficient to propel one to a goal state. But in the absence of some other signal suggesting that one is "getting closer", many seem to override this criterion and interrupt their search.

A factor contributing to this tendency may be the presence of a pair of distinct possible initial steps. The moment that a problem solver begins by filling up one pail, she may already be conscious of the possibility of having made a "wrong" choice, a mistake. The more steps that are taken without reaching the goal, the more likely it 
may seem that a "wrong" move has already been made. Before she takes as many as seven steps, the desire to start afresh can become compelling.

Many trials with no apparent progress can evoke frustration or embarrassment. On the other hand, success in a problem of this sort can be elating-it can reward the engaged problem solver with a feeling of having made a discovery in unfamiliar mathematical territory. This problem also provides a clear opportunity for a student to "look back" after solving it, discovering perhaps that the path she has already found is not the sole solution path. Few problem solvers seem to do this spontaneously. The problem further suggests conjectures as to the general properties of the problem conditions and goal states that permit solutions. In practice few problem solvers spontaneously make such conjectures, or ask questions with respect to possible generalization.

Evidently many problem activities in discrete mathematics can be examined at this level of detail. The discussion of one problem here is intended to serve as a point of reference in asking how experiences in discrete mathematics may provide a basis for developing powerful heuristic processes and powerful affect.

\section{Developing internal systems of representation for mathematical thinking and problem solving}

Let us consider for a moment the ways we have of establishing goals - i.e., setting "instructional objectives" for school mathematics. One may distinguish two different types of objectives; or, perhaps, some may want to consider these as two different levels at which educational objectives may be formulated.

Domain-specific, formal objectives. Here we refer to the desired competencies with conventionally-accepted mathematical definitions, notations, and interpretationsi.e., with shared systems of mathematical representation. These competencies include, but are not limited to, discrete, low-level skills; they can (and should) also include sophisticated methods of solving problems of a variety of pre-established, standard types, and even techniques of proof. Likewise included here are standard mathematical concepts that we expect students to acquire through instruction-i.e., the goal is for our students to construct certain standard internal representations that will enable them to communicate mathematically. Generally speaking, these sorts of educational objectives are highly specific to the particular content domains of mathematics in which they are formulated.

Imagistic, heuristic, and affective objectives. Here we refer to the desired capabilities for mathematical reasoning that enable flexible and insightful problem solving, but are not tied directly to particular notational skills. These goals including the development of powerful imagery, including two- and three-dimensional spatial visualization; the ability to construct new diagrammatic and symbolic representations in non-standard situations; recognition of mathematical structures, and the ability to think structurally; and a highly complex variety of heuristic problem-solving strategies (Polya, 1962, 1965; Schoenfeld, 1983, 1994).
In addition, at this more general level, we include goals that are related to students' affect (McLeod \& Adams, 1989) - not only that they experience the joys of mathematics and satisfaction in mathematical success, but that they be able to bring to mathematical endeavors powerful and appropriate emotional structures that lead to success. In the problem discussed above, for instance, a high expectation of success, anticipation of satisfaction in that success, determination and perseverance, and a readiness to interpret frustration as a signal to be more systematic, can all contribute to increased problem solving powerand our discussion enables us to see why this is so.

In earlier work (e.g. Goldin \& Kaput, 1996; Goldin, 1998), I have proposed a model for mathematical learning and problem solving based on five kinds of internal representational systems:

(a) verbal-syntactic systems associated with natural language;

(b) imagistic systems, including visual/ spatial, auditory/rhythmic, and tactile/ kinesthetic representation;

(c) internalized formal notational systems of mathematics;

(d) a system of planning, monitoring, and executive control that includes heuristic processes; and

(e) affective representation.

These are general human systems, which encode the specifics of mathematical concepts, problems, and solution processes.

The domain-specific, formal objectives suggest a more traditional focus on students' learning of (c), the formal notational systems - the symbolism of mathematics - and methods of symbol-manipulation within such systems; as well as (a), the associated mathematical vocabulary to augment natural language. Such learning is sometimes criticized rather facilely as consisting of rote or meaningless algorithmic processes, but we must note that symbol-configurations and the steps that relate them to each other need not be meaningless just because they are algorithmic, or just because they are predominantly situated within formal notational systems. There is an important difference between abstract mathematical reasoning using formal notations (that interact meaning-fully with other kinds of cognitive representation), and symbol manipulation that is merely decontextualized in the sense that it is detached from meaningful, interpretive representational contexts.

The imagistic, heuristic, and affective objectives imply a focus on developing (b), (d), and (e) powerfully. While these goals are not intrinsically contradictory, and representational systems of different types interact intensively as thinking occurs, it is easy to lose sight of the second set of goals in pursuit of the first. This holds particularly because high-stakes, standardized assess-ments - the "achievement tests" on which school systems in the United States rely so heavily - tend by their nature to focus on the more domain-specific, formal objectives.

Thus, as we consider the uses to make of discrete mathematics in the school curriculum, there is the question of striking an optimal balance between objectives of the two types. Since certain notations and methods of dis- 
crete mathematics are powerful tools in their own right (e.g., combinatorics), some might want to consider these as topics where objectives of the formal type are paramount (e.g., in high school algebra).

Hopefully, few educators would suggest that domainspecific techniques of solving "water pail problems" be included as standard curricular goals in the middle school - though if such problems were included on standardized achievement tests in a misguided effort to assess nonroutine problem solving, we might soon see routine methods for solving them described and applied to many parallel practice problems in school workbooks. Clearly the ideas expressed by DeBellis and Rosenstein, with which this article began, point toward the second type of objectives - those addressing imagistic, executive, and affective representation.

To raise these to paramount status, it becomes important to highlight those specific goals in the development of representational systems where discrete mathematics offers the best possibilities.

\section{A Heuristic Process: Modeling the General on the Particular}

An effective system of planning, monitoring, and executive control for mathematical problem solving does not consist of easy-to-teach components. Rather it develops in the learner over a substantial period of time, through extensive problem solving experiences. But a useful unit of consideration for examining such a developing system is the heuristic process. Heuristic processes refer to complex, partially-defined ways of reasoning that may be given simple names such as "trial and error", "draw a diagram", "think of a simpler problem", and so forth.

In earlier work, Germain and I suggested that heuristic processes involve four dimensions of cognitive analysis (Goldin \& Germain, 1983): (1) advance planning reasons for making use of a particular process, (2) domain-specific methods of applying the process, (3) domains and levels where the process can be applied, and (4) prescriptive criteria suggesting that the process be applied in a given mathematical situation.

One suggestion of this article is that in the discrete mathematical domain, we have a good opportunity to develop the heuristic process "model the general on the particular" in all its ramifications.

Often in discrete mathematics, the problems are quite easy in low-number or small-size cases. In elementary combinatorics, students may solve a problem of permutations or combinations through exploratory methods in a low-number situation, and modeling the general on the particular, frame a conjecture as to the pattern or formula that would apply to a high-number situation. In vertexedge graph theory, students may solve a problem with a graph having only a few vertices and edges, and likewise modeling the general on the particular, frame a conjecture applicable to a category including much larger graphs.

An important point is that in thinking mathematically, we should not stop when the first problem is solved, but should encourage students to learn to ask the generalizing question. Then there is the opportunity to model the general on the particular. Note that this is psychologically quite different from posing the more general problem first, and then trying to use a special case to motivate its solution. In the latter situation, the initial goal is very likely more abstract and daunting. The spontaneous act required is to specialize, and the specialization occurs before the problem has been solved. In modeling the general on the particular, our goal is to reach the point where generalizing questions occur spontaneously to the students. Then they have taken an important step on the way to forming meaningful mathematical abstractions (and also toward what cognitive scientists characterize as "learning transfer").

In the problem of the two pails, we should encourage students to look back after solving it, asking "Are there any other ways of arriving at the goal?" When they have found the alternate solution path, they should be encouraged to invent a variety of generalizing questions; for example, "Can we carry any number of liters of water away from the river (up to the evident maximum of 8)?" "For which pairs of numbers corresponding to the capacities of the pails can such a problem be solved?" "Can we find a property of a given pair of numbers that allows us to solve the problem?" "Is any essential property evident in the problem just solved (i.e., can we make use of the particular)?" "Does anything important change if one pail is much larger than the other, for example pails of 2 and 9 liters respectively (i.e., is the problem example generic)?" "Is there any pattern in the numbers in the solution path cycle?" "How large is the vertex-edge graph containing all the situations that can be reached?" "Is there an interesting generalization afforded by the case where we begin with more than two pails?"

Learning to invent and investigate mathematical questions like these may not immediately improve students' test scores. Nevertheless the payoff in powerful heuristic development, as well as powerful affect, is likely to be substantial. The question, "What makes a problem example a good one for modeling the general on the particular?" leads to the notion of finding the most elementary, generic example; a sophisticated strategy of the research mathematical scientist.

In the problem example given, constructing a non-standard, systematic representation for keeping track of steps proves extremely useful. When we speak of developing the heuristic process "model the general on the particular", we lay the groundwork for its application at more than one domain level. Not only may we model a mathematical approach to a general two-pail problem on the particular ways of generating solution paths that we found in the problem given - we may also model a general strategy of constructing non-standard, systematic representations in nonroutine, high-memory load situations on the particular recognition of the need for such a construction in this problem. That is, problem-solving heuristics (as well as mathematical formulas and concepts) can be abstracted from concrete situations.

\section{Affective Considerations}

I would like to conclude with some additional comments about the opportunities discrete mathematics affords for developing powerful affect (DeBellis \& Goldin, 1997; 
Goldin, 2000). The very phrase "a new beginning" carries with it positive feelings - hope, a sense of freedom from past constraints, and anticipation of new and pleasurable experiences. But will this phrase fulfill its promise over the long run for students and teachers, especially those for whom the affect surrounding mathematics has become painfully negative? To enhance the possibility that it will, we need to understand how discrete mathematical activities can develop specific, empowering affective structures in students.

For example, how do we make good use of the possible feelings of impasse that students may have when confronted with a nonroutine problem? We would like these feelings to result in a sense that "this problem is interesting", and to awaken feelings of curiosity and anticipatory pleasure. Some students may well respond that way; but others may feel nervousness or anxiety. In a small-group problem solving situation, depending on the dynamic, some may experience a sense of inferiority to others. Psychological defenses may be erected at the outset: "I hate these problems, this problem is ridiculous." In discrete mathematics, we have going for us the fact that the situations are often easy to describe and familiar, and early success experiences are not too difficult to build in. When necessary, possible anxiety at the outset can also be relieved by posing the problem situation without the specific goal statement-in the problem of the two pails, by asking simply "What can you do with the pails?" rather than "How can you leave the river with exactly 4 liters?"

"Let's explore!" can be safer than "How can we solve the problem?", because it removes the possibility of failure from the context - allowing more easily for positive meta-affect (see DeBellis \& Goldin, 1997). But our goal should not merely be to reduce anxiety—rather, it should be to provide each student with tools for reducing his own anxiety. If "Here's the situation, what can we do, let's explore!" is experienced as safe, then perhaps the student can learn to respond effectively on other occasions, "This makes me feel anxious—-so I'll just explore!"

We have also seen possible opportunities for developing effective uses of feelings of frustration in students. In the problem of the two pails, we saw how frustration might evoke a desire to give up, or it might evoke a desire to persevere longer in each trial, or to be more systematic in keeping track of previous attempts. It should not be our goal to remove frustration, but to develop the ability in students to channel it into constructive strategic choices - and positive meta-affect, so that the student can "feel good about his frustration" (perhaps because it suggests the problem is especially interesting). Anticipating the frustration, and channeling it toward the adoption of better or different problem-solving strategies, is within reach if it is taken as an explicit goal of discrete mathematical instruction.

In short, we should set out to develop the affect of success through discrete mathematics - the pathways and structures whereby previously unsuccessful students come to feel, "I am really somebody when I do mathematics like this!"

\section{References}

DeBellis, V. A., \& Rosenstein, J. G. (2004). Discrete mathematics in primary and secondary schools in the United States. ZDM (this volume).

DeBellis, V. A. \& Goldin, G. A. (1997). The affective domain in mathematical problem solving. In E. Pehkonen (Ed.), Proceedings of the 21st Conference of the International Group for the Psychology of Mathematics Education, Lahti, Finland (Vol. 2, pp. 209-216). Helsinki: University of Helsinki Department of Teacher Education.

Gardiner, A. D. (1991). A cautionary note. In M. J. Kenney \& C. R. Hirsch (Eds.), Discrete Mathematics Across the Curriculum, K-12. Reston, VA.: National Council of Teachers of Mathematics.

Goldin, G. A. (1998). Representational systems, learning, and problem solving in mathematics. Journal of Mathematical Behavior 17(2), 137-165.

Goldin, G. A. (2000). Affective pathways and representation in mathematical problem solving. Mathematical Thinking and Learning 2(3), 209-219.

Goldin, G. A. (1984). Structure variables in problem solving. In G. A. Goldin \& C. E. McClintock (Eds.), Task Variables in Mathematical Problem Solving (pp. 103-169). Philadelphia, PA: Franklin Institute Press (now Hillsdale, NJ: Erlbaum),.

Goldin, G. A., \& Germain, Y. (1983). The analysis of a heuristic process: 'Think of a simpler problem'. In J. C. Bergeron \& N. Herscovics (Eds.), Proceedings of the 5th Annual Meeting of $P M E-N A$ [North American Chapter, International Group for the Psychology of Mathematics Education] (Vol. 2, pp. 121128). Montreal: Univ. of Montreal Faculty of Educational Sciences, pp. 121-128.

Goldin, G. A., \& Kaput, J. J. (1996). A joint perspective on the idea of representation in learning and doing mathematics. In L. Steffe, P. Nesher, P. Cobb, G. A. Goldin, and B. Greer (Eds.), Theories of Mathematical Learning (pp. 397-430). Hillsdale, NJ: Erlbaum.

Kenney, M. J. \& Hirsch, C. R. (Eds.) (1991). Discrete Mathematics Across the Curriculum, K-12. Reston, VA.: National Council of Teachers of Mathematics.

McLeod, D. B., \& Adams, V. M. (Eds.) (1989). Affect and Mathematical Problem Solving: A New Perspective. New York: Springer Verlag.

Polya, G. (1962, 1965). Mathematical Discovery: On Understanding, Learning, and Teaching Problem Solving, Vol. I and Vol. II. New York: John Wiley \& Sons.

Rosenstein, J. G., Franzblau, D., \& Roberts, F. (Eds.) (1997). Discrete Mathematics in the Schools. Washington, DC: American Mathematical Society, and Reston, VA: National Council of Teachers of Mathematics.

Schoenfeld, A. H. (1983). Episodes and executive decisions in mathematical problem-solving. In R. Lesh \& M. Landau (Eds.), Acquisition of Mathematics Concepts and Processes (pp. 345-395). New York: Academic Press.

Schoenfeld, A. H. (Ed.) (1994). Mathematical Thinking and Problem Solving. Hillsdale, NJ: Erlbaum.

\section{Author}

Goldin, Gerald A., Prof., Center for Mathematics, Science, and Computer Education, Rutgers University, 118 Frelinghuysen Road, Piscataway, NJ 08854, USA..

Email: gagoldin@dimacs.rutgers.edu 